\title{
Quaderni
}

QUADERNI Communication, technologies, pouvoir

71 | Hiver 2009-2010

Le changement climatique : les résistances à

l'adaptation

\section{L'inscription du projet de Métropole de Paris dans l'espace public numérique : émergences et ouvertures}

\section{Dominique Pagès}

\section{(2) OpenEdition \\ Journals}

Édition électronique

URL : http://journals.openedition.org/quaderni/531

DOI : 10.4000/quaderni.531

ISSN : 2105-2956

Éditeur

Les éditions de la Maison des sciences de l'Homme

Édition imprimée

Date de publication : 5 janvier 2010

Pagination : 75-84

\section{Référence électronique}

Dominique Pagès, «L'inscription du projet de Métropole de Paris dans l'espace public numérique:

émergences et ouvertures ", Quaderni [En ligne], 71 | Hiver 2009-2010, mis en ligne le 05 janvier 2012, consulté le 22 avril 2019. URL : http://journals.openedition.org/quaderni/531 ; DOI : 10.4000/ quaderni.531 


\section{Politique}

\section{l'inscription du projet de Métropole de Paris dans l'espace public numérique : émergences et ouvertures}

Le projet de loi sur le Grand Paris a été adopté de $1^{\text {er }}$ décembre 2009 en première lecture par l'Assemblée nationale après avoir été discuté pendant plus d'une semaine. Ce vote marque une accélération du processus de métropolisation tant en termes politiques, urbanistiques que médiatiques.

Depuis la naissance de la Conférence métropolitaine en juin 2006 et la création de son site en juin 2008, inscrits dans la continuité de l'action de la Mairie de Paris et plus particulièrement de celle, de longue haleine, de l'élu délégué depuis 2001 au projet Paris Métropole (Pierre Mansat), une diversité de sites publics, politiques, experts et citoyens ont éclos, voués à l'explicitation, l'illustration et à la mise en débat du projet de métropolisation de Paris. Chacun propose un angle de vue plus ou moins structuré, donne une vision du territoire métropolitain plus ou moins compréhensible, traduit des formes et des valeurs plus ou moins explicites. Chacun écrit le projet et le processus à sa manière, utilisant de manière plus ou moins opportune les ressources du web.

Derniers nés, marquant l'actuelle montée en puis-

Dominique Pagès

Maître de Conférences CELSA sance de l'offensive, le site « Mon Grand Paris » (lancé en novembre par le Service d'Information du Gouvernement) qui se dit « dédié au citoyen » (www.mongrandparis.fr), et le site refondu du Syndicat Métropole (www.paris.metropole, l'ancien site était un héritage de la conférence métropolitaine) qui s'est ouvert officiellement le 17 décembre.

Ces propositions numériques, relayées par les médias en ligne et par une diversité d'acteurs publics et anonymes, représente aujourd'hui un 
ensemble assez complexe à déchiffrer. Cet article se propose de clarifier cette inscription du projet métropolitain dans l'espace numérique afin de poser les bases d'une réflexion à venir plus approfondie sur la mise en récit de la métropole de Paris. Il s'agit de faire un point relativement descriptif sur la présence numérique du projet de Paris Métropole et donc sur la diversité de ses principaux énonciateurs.

\section{Brève généalogie de la communication publique autour projet}

Ce mouvement éditorial portant sur la Métropolisation de Paris fut amorcé par la délégation à la coopération territoriale et est donc lié à la stratégie d'un élu " évangélisateur » (l'adjoint au maire de Paris délégué à Paris Métropole) et de son équipe.

La création de cette délégation inédite fut en effet à l'origine une intense activité tant relationnelle, intellectuelle qu'éditoriale: ainsi en octobre 2001 fut créée La Lettre de Coopération territoriale " Extramuros » (dont le dernier numéro devrait paraître début 2010) ${ }^{1}$; en 2002 , une exposition «Territoires partagés, l'archipel métropolitain », organisée par l'Arsenal, sensibilisa le monde des architectes; de 2002 à 2008, les actions se multiplièrent visant avant tout à susciter l'effervescence intellectuelle autour des questions de métropolisation : des table-rondes, des sémi naires, des colloques d'experts puis en juin 2006, la création de la Conférence Métropolitaine qui se transforma en juin 2008 en Assises de la Métropole ; début 2009, le lancement du Syndicat mixte Paris Métropole fut en partie une réponse politique à la nomination d'un secrétaire d'État,
Christian Blanc, au développement de la Région Capitale.

Les publications de l'APUR (l'atelier parisien d'urbanisme), de l'Arsenal, de l'IAURIF (L'Institut d'Aménagement de la Région Ile de France) et les travaux d'une diversité d'acteurs et experts métropolitains (ainsi les rapports Dallier ${ }^{2}$, Planchou) amplifièrent cette médiatisation du processus de métropolisation par la Mairie de Paris $^{3}$. Cet ensemble quelque peu décousu et relativement confidentiel permit d'appuyer la constitution d'un réseau de recherches, de faire émerger des thématiques fortes et de donner une visibilité au projet.

Nous nous centrerons ici sur les sites internet et tout d'abord sur ceux qui ont émergé en fonction de faits et d'échéances politiques : la montée en puissance du projet Paris Métropole (2006-2008) ; l'affirmation des experts dans cette montée (2007-2009) ; le lancement du secrétariat d'État puis du Syndicat Paris métropole (juin 2008 puis janvier 2009) ; le lancement de la consultation des architectes (2008) et l'actuelle radicalisation des projets (juin-décembre 2009). Pour ce faire trois entrées sur Google ont été retenues : « Métropole de Paris », « Paris métropole », « Grand Paris ».

\section{Des sites institutionnels : de la première heure au dernier venu...}

Le site de la Mairie de Paris (www.paris.fr) a ouvert une rubrique dès 2006 pour faire comprendre l'avancée du projet, des négociations, des projets et donner tous les éléments de connaissance nécessaire à sa compréhension (rubriques actuel- 
les : actualités, acteurs et partenaires, coopération sur le territoire, coopération par thème, etc.). Cette entrée Paris Métropole (à plusieurs reprises refondue) est un centre de ressources conséquent et s'inscrit dans une visée institutionnelle. Depuis plus d'une année, il s'est ouvert à une consultation par les internautes élargie, notamment en éditorialisant plus clairement ses contenus et en intégrant des vidéos d'habitants interrogés sur la question « métropole », sur leur vision, leurs attentes (« Paroles de métropolitain(e)s »).

Le Conseil régional s'est très tôt positionné via son site sur le projet (www.iledefrance.fr : l'auteur en est l'équipe élue). Il vise à faire de ce sujet un débat public, mais sans ouvrir à une possible interactivité. Il s'est d'abord proposé de raconter et d'illustrer l'histoire, de camper le cheminement du projet en valorisant le citoyen comme un acteur de ce mouvement. Aujourd'hui, la démarche va plus loin puisqu'il édite et commente les « scénarii pour la métropole », issus du rapport remis par une commission d'élus. Mais la posture reste paradoxale car le projet métropolitain met en question les frontières et la légitimité de l'institution régionale.

Le site « le Grand Pari(s) de l'Agglomération parisienne » (www.legrandparis.culture.gouv. fr), lancé en 2008 par le ministère de la Culture à l'occasion de la consultation du ministère de la Culture et de la Communication, s'affiche lui aussi comme institutionnel: on peut y lire des auteurs de la majorité (Albanel et Blanc) comme de l'opposition (Hidalgo et Ferri), y écouter des interviews de personnalités concernées par le projet, y accéder à « la lecture transversale de la consultation au travers de dossiers thématiques ».
Le ton relativement laudatif et enthousiaste, l'intégration de vidéos témoignent d'une volonté plus grande que le site précédent de susciter l'imaginaire, d'évoquer la démarche innovante et inspirée. Mais sa visée est bien politique, en ce qu'il constitue le premier jalon d'une stratégie médiatique offensive (celle de la consultation puis de l'exposition de ses propositions à la Cité de l'architecture et du patrimoine, celle de l'actuelle consultation). Visuellement, la thématique métropolitaine de la « polycentralité » est traduite par des jeux de cercles imbriqués.

Le site proposé depuis novembre 2009 par le Service d'Information du gouvernement « Mon Grand Paris » (www.mongrandparis.fr), reprend mais en s'en démarquant les codes graphiques du précédent. À partir d'un slogan simple « une idée qui a besoin des vôtres », ce site appelle les Franciliens et les Français en général à apporter leur avis et témoignages, leurs suggestions et propositions et enfin « leur bonne volonté » au projet du Grand Paris; et ceci autour de 5 thèmes (ville et campagne, quartiers et logement, gare et transports, développement économique et culture). Une vidéo met en scène des dialogues enjoués et scénarisés sur le projet métropolitain. Un agenda propose le calendrier des « rencontres territoriales » organisées du 3 au 10 décembre, ouvertes au grand public et réunissant quelques acteurs du projet « la Métropole de demain ». On peut suivre les contributions qui sont analysées par un rapporteur Internet dont la mission est de permettre de « faire émerger des propositions concrètes ». Une campagne plurimédias (notamment radiophonique) accompagne son lancement.

Ces sites sont encore inégalement relayés par 
ceux des collectivités territoriales franciliennes dont la plupart se cantonnent à une simple voire très discrète information sur le processus dans lequel nombre d'entre elles se sont pourtant inscrites. Le nouveau site du Syndicat Paris Métropole lancé fin décembre devra jouer le rôle de relais et d'incitateur auprès des communes, départements et acteurs publics métropolitains.

\section{Les sites politiques : les pérennes et les éphémères}

Le site de Pierre Mansat (www.pierremansat. fr) fut bien sûr le premier à présenter, illustrer, commenter le projet dès 2007 (après avoir tenu un blog intitulé « l'autre Paris », lautreparis. overblog.fr). Il se présente aujourd'hui comme un blog incontournable, même si l'interactivité y est réduite. Des billets et des dossiers thématiques actualisés ponctuent l'agenda du projet métropolitain, en précisent en continu les enjeux et les actes, en commentent les évolutions, tout en relayant les travaux experts ou les regards de portée sociale. La volonté de convaincre est forte, mais il s'agit plus de transmettre que d'échanger, de donner des éléments pour s'informer et se forger une opinion mais aussi un regard (présence affirmée de photographies). Au fil du temps la présence de l'élu s'est affirmée, dans le ton et dans la forme, en réaction sans doute à la concurrence croissante d'énonciateurs divers. Si ce site-blog reste avant tout un centre ressources de référence pour les experts, les chercheurs, les commentateurs voulant déchiffrer la métropolisation de Paris en temps réel son audience et sa portée se sont considérablement accrues depuis 2008.

Dans le mouvement de la campagne municipale de 2008 divers sites se sont centrés sur la question métropolitaine ou ont été créés à cet effet. On évoquera les plus offensifs: celui de Roger Karoutchi (le blog http://www.debat-grandparis. com/) et celui du parti du nouveau Centre, intitulé « Grand Paris 2014 » (www.grandparis.2014. com). Ce dernier se veut collaboratif, «fédérant toutes les énergies qui se proposent d'y participer » et se présente comme « un outil d'échange permanent, installé dans la durée, entre élus du Nouveau Centre et 'grands parisiens de cour' qui nous feront ici des suggestions, nous donneront des idées pour avancer vers le 'Grand Paris'». Aujourd'hui quelque peu en sommeil, il se propose pourtant de poursuivre le débat en demandant aux internautes avis, opinions « pour que la mandature 2008-2014 soit utile», accompagnant l'évolution du projet territorial.

La campagne desRégionales à venir voit de même apparaître des initiatives métropolitaines partisanes: ainsi la création de la structure dénommée Ile de France Métropole (association domiciliée à Roissy) sous l'impulsion de Patrick Devedjian, qui n'a pas encore de site mais dont la naissance et les objectifs (une plateforme de réflexion autour des grands projets et de leur concertation) sont relayés par celui du Conseil Général des Hauts de Seine.

\section{Des sites d'experts à faible ouverture interdisciplinaire}

Acteur incontournable de l'aménagement métropolitain, l'IAU (l'Institut de l'Aménagement et de l'Urbanisme en Ile de France : www.iau-idf. fr), dont les membres du Conseil d'administration émanent de diverses structures représentant 
du Conseil Régional, Préfet de la région, un représentant de l'INSEE, etc., met à disposition sur son site de nombreux contenus (notamment visuels) concernant plus ou moins directement les projets d'aménagement et d'urbanisme de la région métropolitaine. Ce site vise la clarification, l'exhaustivité, mais s'adresse avant tout à des personnes « concernées ». La présentation du projet est fondée avant tout sur une vision « moderne » de la ville, témoignant d'une transformation interne majeure des infrastructures et des moyens de communication.

Le blog « Le Grand Paris des urbanistes » ouvert en 2008 ( http://planningforparis.blogspot.com) est quant à lui édité par des urbanistes qui appartiennent au réseau de l'Institut d'Urbanisme de Paris. Les auteurs y affirment un regard expert sur la «complexité métropolitaine », veulent y « exprimer le point de vue d'une profession sur un projet politique », apporter « un regard averti » et se positionner comme "une valeur ajoutée au débat tout en commentant l'actualité » via des commentaires d'articles mis en ligne et des renvois à des études d'aménagement. Les images métropolitaines se multiplient, des tentes de sdf sur les quais parisiens à l'incontournable vue d'avion.

Le site Métro-pole (www.metro-pole.net) se présente quant à lui comme « un site d'information indépendant des entreprises de transport et des collectivités locales ». Un site indépendant (du STIF, des entreprises de transports et des collectivités territoriales) qui se définit comme collaboratif et réalisé « par des professionnels et des passionnés de transports publics franciliens ». Un site qui se focalise sur les transports métropo- litains (actualité, réseaux et lignes, informations techniques, photothèque, portraits et carnets du voyageur, etc.), défendant le transport comme un élément structurant de la métropolisation et souhaitant «faciliter ses usages », notamment via un forum. Malgré ses intentions affirmées et les services offerts, les choix visuels de ce site restent très parisiens ${ }^{4}$.

On peut évoquer ici la page Wikipédia actuellement en cours de réécriture, passant d'une approche généraliste et généalogique à une approche plus liée à l'agenda actuel. Cette page « Paris Métropole » visant le grand public consiste encore en un résumé historique, une présentation des enjeux et des débats actuels, en une évocation des tensions politiques autour de la construction de la métropole. Son choix photographique pose question: les deux photographies choisies illustrent les travaux de couverture du périphérique à la Porte des Lilas. L'imaginaire construit reste donc celui de la cicatrisation de la rupture entre le centre et la banlieue de Paris.

\section{Des sites alternatifs et citoyens hétérogènes}

Cet exposé ne saurait minorer des sites plus marginaux mais dont la persistance et la présence médiatiques (visibilité de l'auteur, qualité du référencement, interpellation de l'internaute...) témoignent d'une place singulière dans cette énonciation métropolitaine.

Le blog Paris Galaxie (http://parisgalaxie.blogspot.com) se présente comme « un laboratoire d'ingénierie d'idées, de recherche, et de création confidentielle, développé depuis $2000 »$ comme «un scénario alternatif et non conventionnel pour 
l'agglomération parisienne ». Il affirme son indépendance et propose des pistes pour construire la métropole, démarche à la fois " prospective et pratique ». Mais son auteur, Raphaëlle BidaultWaddington, est tout à la fois artiste et membre du Modem (elle fut tête de liste pour le 3ème arrondissement). Visuellement, le site propose de singulières projections numériques traduisant d'aussi singuliers scenarii métropolitains.

Le blog « Paris est sa banlieue » (http://parisbanlieue.blog.lemonde.fr) suit le débat depuis son origine voulant tout à la fois informer au plus près et au plus vite, tout en prenant ouvertement parti. Il intègre des interviews vidéos d'acteurs centraux du projet métropolitain. Son auteur, Jean Paul Chapon, y livre des billets d'humeur sur la vie politique, la société et la culture francilienne et semble donc reconnu par les protagonistes comme un relais d'opinion légitime.

\section{Sur les marges, des sites d'acteurs marchands et culturels}

On constate l'existence de sites concernés plus par le territoire culturel élargi de la capitale que par l'actuel débat politique, urbanistique et citoyen.

Ainsi le blog « Métropole de Paris » (www.metropoleparis.com, créé en de 1996) propose en fait «a weekly online magazine, for lovers, dreamers, topless romantics and ordinary visitors to Paris past, present, futur, virtual». Ce site, proposé par un amoureux de la capitale, ne déborde guère de ses frontières.

Le site du Comité régional du tourisme dépendant du Conseil Régional est plus intéressant par sa lecture métropolitaine du territoire francilien. Il tente depuis deux années d'élaborer une vision renouvelée de la destination Paris: à la fois plus actuelle, plus évènementielle et débordant largement, dans ses incitations de visites, les frontières géographiques de la Capitale (www.pidf.fr). La région à la conquête de la capitale : ce mouvement est bien celui d'une stratégie symbolique métropolitaine, aussi contestables en soient les choix visuels.

Dans cette catégorie, on peut intégrer les sites éphémères d'évènements métropolitains (Nuits Blanches, Futurs en Seine, Festival de l'ô) qui ont récemment accompagné ce mouvement émergent d'écriture culturelle, artistique et touristique de la métropole de Paris.

Enfin les sites d'opérateurs des transports ouvrent à des représentations inédites, voire plus proches des plus jeunes (RATP et ses campagnes : celle de 2002 signée Nan Goldin ou celle de 2009 « Imagin-R », www.imagine-r.com).

\section{Les médias généralistes: des relais actifs ?}

Les médias généralistes ont inégalement relayé sur Internet l'actualité métropolitaine ces deux dernières années. De Rue 89 à 20 minutes (qui a créé une rubrique sur le Grand Paris), du Nouvel Observateur (qui fit de même) à l'Express, se sont multipliés articles, tribunes, analyses, tentant de suivre le déroulé du récit métropolitain ou plutôt de ses fragments ${ }^{5}$, au risque parfois de confondre urgence et prospective, détails et grandes perspectives, et de résumer ce projet à à des rivalités politiques, voire de personnes, et donc à une 
temporalité politique. La diversité des formes de traitement médiatique du projet de la métropole de Paris mériterait une étude plus approfondie ${ }^{6}$. Dans l'attente de celle-ci, rendons simplement hommage au site « la vie des idées » (www.laviedesidees.fr, magazine international d'analyses et d'information) qui a pris pleinement part à la discussion du projet en relayant notamment l'avis de professionnels de la question territoriale (Frédéric Gilli, Laurent Davezies entre autre) mais aussi d'internautes impliqués (conseillers municipaux, grands témoins, etc.). Plus qu'un site relais d'opinion, ce magazine « on line» d'analyses et d'informations interdisciplinaires se veut non partisan. Son intention est réflexive et distanciée: il s'agit de mettre à disposition des savoirs, des contenus, des analyses produits par des sources légitimes. Le ton est quelque peu académique et le style avant tout analytique. Il n'y a pas de place pour l'échange et le débat.

\section{Un traitement médiatique numérique en devenir}

$\mathrm{Au}$ final, cet ensemble composite et en voie de repolitisation conjoncturelle (les élections régionales) nous dit la nécessité d'une réflexion " communicationnelle » sur le projet métropolitain. Celui-ci s'est longtemps écrit en termes technocratiques et experts (essentiellement d'aménagement), puis il s'est affirmé de manière spectaculaire au fil des propositions des urbanistes et des architectes; enfin il s'est plus récemment revendiqué en termes de lutte partisane de grande amplitude (notamment dans la perspective des régionales) plus qu'en un projet de développement territorial, sociétal, social et culturel.
Visuellement, le projet se traduit au fil de ses sites par des représentations contradictoires: tour à tour abstraites (des cartes de tous genres, des schémas des cercles et des flèches voulant traduire le décentrement, la polynucléarité, l'absence de frontière); plus strictement géographiques et urbanistiques (omniprésence des « vues d'en haut », marquant une position de surplomb ); utopiques et spectaculaires (le foisonnement des imageries proposées par les dix équipes mais aussi les représentations récurrentes de "skylines " ponctuant une diversité d'ouvrages, invitations à des expositions, de blogs $)^{7}$.

Cette profusion visuelle rend difficile la compréhension intime et l'appropriation réfléchie d'un possible territoire, ouvert et à géométrie variable. On y cherche avec difficulté un regard d'ensemble porté sur les espaces vécus et leur transformation, sur les modes d'habiter et de se déplacer «métropolitains », sur les réalisations effectives et à venir. Du quotidien métropolitain actuel et futur, les images ne nous disent que peu ${ }^{8}$.

De même, textuellement, on ne trouve guère sur les sites les plus grand public de définition de « la métropole », assumée en termes historiques, politiques, sociologiques et culturels. La définition de l'objet même de ces sites est le plus souvent allusive ou superficielle, alors qu'elle devrait en constituer le point de départ et la finalité.

Une métropole, rappelons-le, c'est un territoire monde, sans frontière tangible, à la gouvernance souvent improbable, qui doit pourtant fédérer autour d'elle (en France) des banlieues dont certaines sont dites sensibles, des villes patrimoniales, des départements, des territoires périphé- 
riques et des zones périurbaines, des territoires intercommunaux.

Les métropoles, ce sont ces formes de collectivité « post-urbaine » qui traduisent les valeurs, les aspirations et les contradictions de la globalisation, articulant les échelles locales et globales dans une perspective concurrentielle tant économique que culturelle ; elles visent et cherchent en effet à s'inscrire de manière créative dans un jeu concurrentiel mondial. Ce sont dans les pays riches ces grandes agglomérations dites mobiles et flexibles vouées au culte de l'innovation et qui dominent et concentrent la puissance économique, financière, technologique ainsi que les ressources en main d'œuvre qualifiée et diversifiée (... et ce sont dans les pays pauvres ces territoires souvent sans planification qui concentrent la pauvreté dans tous ses états).

Les métropoles, ce sont ces systèmes sociaux et spatiaux qui ne peuvent exister que par des réseaux permettant la mobilité et l'émergence d'une pluralité de centres ; ce sont ces nouvelles formes de vivre ensemble oscillant entre proximité et mobilité, entre quête d'individuation et recherche du collectif surtout s'il est éphémère, entre soi et altérité ; ce sont ces espaces temps, ces rythmes qui sont très liés aux nouvelles technologies de la communication mais aussi à la place renouvelée des espaces publics, ceux-ci devant plus qu'auparavant sans doute affirmer de nouvelles cohérences territoriales ${ }^{9}$.

Cette amorce de définition ouverte permet de situer l'enjeu civilisationnel des processus en cours et aimerait inciter à un travail de conceptualisation préliminaire à toute traduction numérique.
Aujourd'hui le processus de métropolisation semble être devenu une rumeur médiatique pour le grand public. Pour fonder une dynamique de longue haleine, une approche théorique et symbolique de plus grande ampleur, évolutive et interdisciplinaire est nécessaire. Les Sciences de l'information et de la communication y sont centrales, tant les technologies de communication sont constitutives du fait métropolitain. Au creux de ces enjeux de communication et de médiatisation, se posent en effet les questions d'une identité métropolitaine et d'un modèle de vivre ensemble. 
$\mathrm{N} \cdot \mathrm{O} \cdot \mathrm{T} \cdot \mathrm{E} \cdot \mathrm{S}$

1. Cette lettre se veut être un outil de dialogue et d'information destiné à tous les acteurs franciliens de la coopération métropolitaine mais aussi un espace de réflexion et de prospective.

2. Philippe Dallier ouvrit un blog (blogsenat. fr.grandparis, aujourd'hui fermé) qui exposa les travaux menés par le sénateur pour l'Observatoire de la décentralisation du Sénat en vue de proposer un projet global cohérent pour la métropole parisienne.

3. Ainsi dernièrement : le $\mathrm{n}^{\circ} 38$ de la Revue Paris Projet intitulé « Regards croisés : métropoles Européennes » et le $n^{\circ} 39$, « une petite synthèse du Grand Paris de l'Agglomération parisienne ».

4. On peut aussi citer dans ce registre le « blogencommun.fr » qui se présente comme un blog collaboratif sur les transports en communs en Ile de France, indépendant des régies de transports et se proposant de raconter « vos histoires et anecdotes sur votre ligne de métro, de RER, de bus ».

5. Voir le numéro 4 de la Revue d'Architecture dont le dossier porte sur les « Fragments métropolitains».

6. À titre d'exemples du suivi médiatique de la bataille politique, citons deux titres emblématiques : « Grand Paris, petits calculs politiques ", La Tribune, 10 octobre 2007 ; «Le Conseil régional Ile de France tente de neutraliser le projet de Grand Paris », Les Échos, 4 janvier 2008.

7. Ainsi l'exposition « L'invention de la Tour Européenne » au pavillon de l'Arsenal (mai-octobre 2009) qui interroge la pertinence d'une spécificité associée à l'architecture verticale; celle sur « la Consultation des architectes sur l'avenir du Grand Paris » à la Cité de l'Architecture et du Patrimoine ; mais aussi la couverture de Télérama d'avril 2008, etc.
8. Voir pour cela l'exposition «Villes Rêvées, villes durables » à l'espace Electra de la fondation EDF et plus spécifiquement la salle "Constellations » qui visualise des formes individuelles concrètes de mobilités métropolitaines (23 octobre 2009 - 7 mars 2010).

9. Ainsi le système renouvelé des espaces verts et du maillage des lieux publics, des gares centrales et périphériques, des aéroports locaux ou internationaux, etc. 
$R \cdot E ́ \cdot S \cdot U \cdot M \cdot E ́$

La métropolisation de Paris est entrée depuis 2008 dans un moment politique complexe et conflictuel, tant en termes de gouvernance, de choix architecturaux et urbanistiques que de médiatisation. C'est cette inscription du projet de la Métropole de Paris dans l'espace public et politique qu' analyse cet article. La diversité des actuels énonciateurs et l'hétérogénéité de leur vision (Paris Métropole, Grand Paris, Région Métropolitaine, etc.) y sont prises en compte au fil de la lecture des sites Internet « métropolitains » qui ont émergé entre 2006 et 2009. De quelle métropole parle-t-on et comment ? En quoi Internet est-il à la fois une caisse de résonance idéologique et symbolique de ce « Grand Projet »? L'auteur s'efforce ici de clarifier cet enchevêtrement de points de vue et de représentations du projet métropolitain afin d'ébaucher une réflexion critique à venir sur l'écriture médiatique des métropoles.

\section{Summary}

Paris conurbation since 2008 came at a time and complex political conflict, both in terms of governance, urban and architectural choices that mediatization. It is this inclusion of the Metropole Paris project in public and political space which is discussed in this article. The diversity of current enunciators and heterogeneity of their vision are taken into account in the course of reading the websites "metropolitan" that emerged between 2006 and 2009. In what city does this on and how? What is the Internet both a ideological and symbolic sounding of this "big project"? The author here attempts to clarify the tangle of points of view and representations of the metropolitan project to outline a critical reflection on the future of this mediatization. 